\title{
New spirocyclic nitroxides of 2,5-dihydroimidazole series flanked by two mesogenic fragments
}

\author{
Elena V. Zaytseva, ${ }^{a *}$ Andrey V. Shernyukov, ${ }^{a}$ Alexander M. Genaev, ${ }^{a}$ Rui Tamura, ${ }^{b}$ Igor \\ A. Grigor'ev, ${ }^{\text {a }}$ and Dmitrii G. Mazhukin ${ }^{\mathrm{a}, \mathrm{c}_{*}}$ \\ ${ }^{a}$ N.N. Vorozhtsov Novosibirsk Institute of Organic Chemistry of the Siberian Branch of Russian \\ Academy of Sciences, ave. Acad. Lavrent'eva 9, Novosibirsk, 630090, Russia, \\ ${ }^{b}$ Graduate School of Human and Environmental Studies, Kyoto University, Yoshida-nihonmatsu- \\ cho, Sakyo-ku, Kyoto 606-8501, Japan \\ ${ }^{c}$ Novosibirsk State University, Pirogova Str. 2, Novosibirsk, 630090, Russia \\ E-mail:elena@nioch.nsc.ru, d-mazhukin@yandex.ru
}

DOI: http://dx.doi.org/10.3998/ark.5550190.p008.808

\begin{abstract}
$\mathrm{N} / \mathrm{C}$-Hydroxylated spirofused derivatives of 2,5-dihydroimidazole which were synthesized by condensation of 4-(4-hydroxyphenyl)cyclohexanone with aryl hydroxylaminoalkyl ketones in the presence of ammonia were determined to be trans-ee-isomers of 1,4-cyclohexane framework by NMR spectroscopy. Oxidation of the cyclic hydroxylamino moiety followed by acylation of the phenolic hydroxyl group with 4-alkyloxybenzoic acid chlorides led to the corresponding spirocyclic nitroxides bearing two mesogenic fragments.
\end{abstract}

Keywords: Nitroxides, free radicals, 2,5-dihydroimidazoles, 2-hydroxylamino ketones, mesogens

\section{Introduction}

The rapid development of synthetic chemistry of stable nitroxyl radicals (aminoxyls) has led to significant advances in the field of their practical importance. They have proven to serve as (i) very effective stoichiometric and catalytic oxidants for alcohols, enolates, electron-rich olefins, carbanions, etc., ${ }^{1,2}$ (ii) key agents for nitroxide-mediated radical polymerization, ${ }^{1}$ and (iii) structural units for creating single molecular magnets ${ }^{3}$ and ferromagnetics. ${ }^{4}$ Another aspect of their use is the creation of advanced paramagnetic liquid crystalline (LC) materials based on them. ${ }^{5}$ In fact, it attracted a great deal of attention because the obtained materials could exhibit unique magnetic interactions and unconventional magneto-electric or magneto-optical properties. ${ }^{6}$ Over the last decade a number of calamitic all-organic chiral PROXYL-type LC 
radicals 1 were developed and their magnetic properties have been thoroughly investigated. ${ }^{7-10}$ This type of organic radicals has shown a set of prominent physical properties in the LC state such as generation of a sort of spin glass-like inhomogeneous ferromagnetic interactions under weak magnetic fields, ${ }^{9,10}$ possibility of the second harmonic generation, ${ }^{11}$ and existence of two bistable states in ferroelectric LC phase. ${ }^{12,13}$

In connection with these aforesaid studies, it seems to be promising to synthesize a new type of all-organic paramagnetic LC materials bearing a nitroxide fragment in heterocyclic core such as 2,5-dihydroimidazole. Insertion of the second imine type of nitrogen atom being effective binding site can provide additional features and advantages in development of new soft materials, such as $\mathrm{pH}$-sensitive LC droplets ${ }^{14}$ or self-organized nanoparticles in LC medium. ${ }^{15,16}$ The introduction of a spirocyclic fragment as a building block into the molecule structure has been a subject of considerable interest. Tschierske and Vögtle et al. succeed in the synthesis of a number of calamitic LCs on the basis of mono- and dispiro derivatives of cyclobutanes, cyclopentanes and cyclohexanes. ${ }^{17-22}$ Interesting results were obtained in the preparation ferroelectric LC's on the basis of triangular structures $(3+3+3)^{23,24}$ and gem-difluoro derivatives of spirocyclopropanes $(3+6+3),{ }^{25}$ which showed chiral smectic $\mathrm{SmA}^{*}$ and $\mathrm{SmC}^{*}$ phases in different conditions.

This report is a continuation of the researches on the synthesis of spirocyclic mono- and biradicals of azole series which can show mesogenic properties ${ }^{26-28}$ and serve as spin probes for diamagnetic LCs by ESR spectroscopy. ${ }^{29,30}$ In this work we describe an approach to the synthesis of stable spirocyclic nitroxides 2-4 with a 2,5-dihydroimidazole structure (Figure 1).

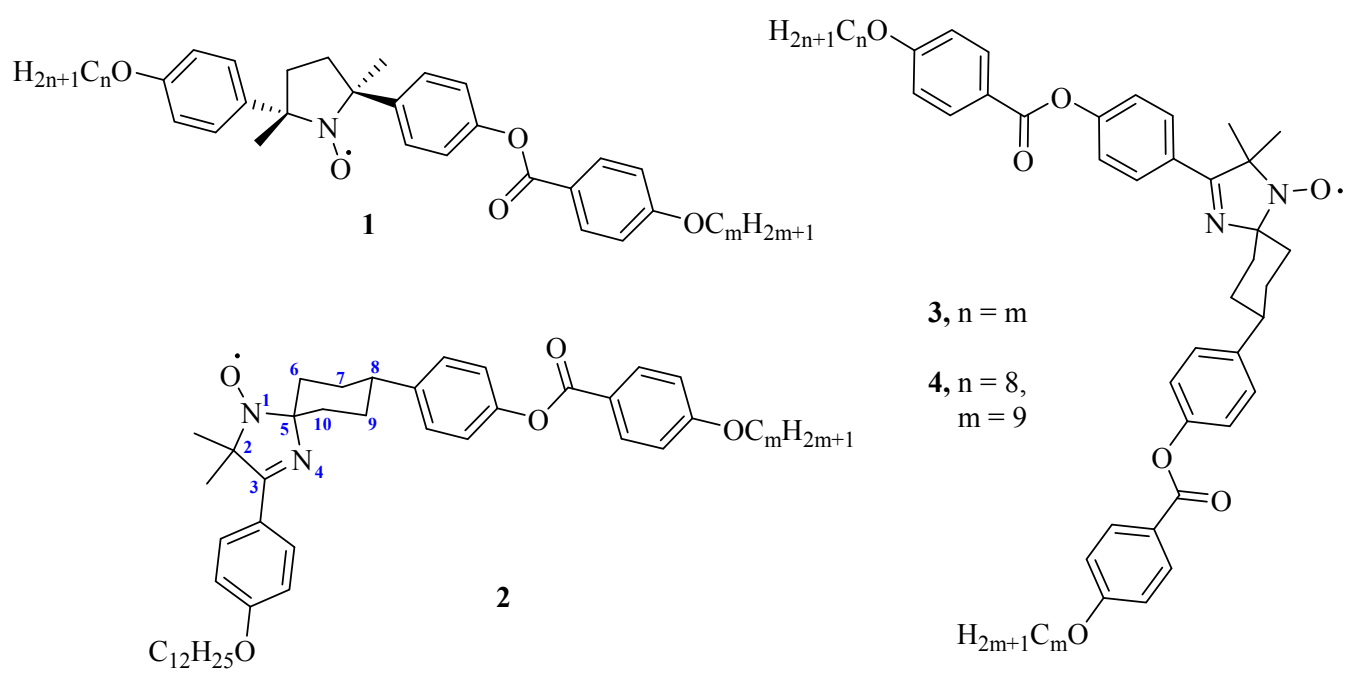

Figure 1. Compounds 1 - Pyrrolidine-type all-organic LC nitroxides (PROXYLs).Compounds 2-4 - target 2,5-dihydroimidazole-type spirocyclic nitroxides. 


\section{Results and Discussion}

Despite the fact that a great number of LC molecules are often not ideal calamitics and have bulky substitutients near the lateral and terminal atoms, ${ }^{31}$ we have tried to choose for our investigation such model compounds which satisfied the requirement of rod-like configuration. First, we performed the molecular modeling (see Experimental) for the structure with heterocyclic 2,5-dihydroimidazole core spirofused with cyclohexane moiety possessing 4(aroyloxy)phenyl substituent on the $\mathrm{C} 4$ position. Analysis of obtained data for model diastereomers $\mathbf{A}$ and $\mathbf{B}$ revealed that $\mathbf{A}$ is $\mathrm{Z}$-shaped while $\mathbf{B}$ can meet the requirement to be rodlike molecule (Figure 2).

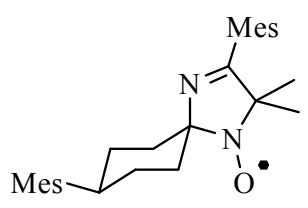

A

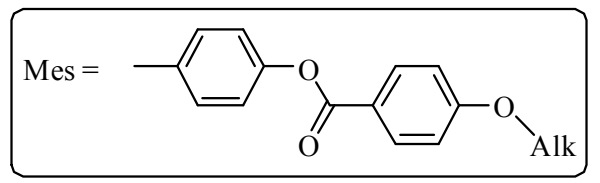

B
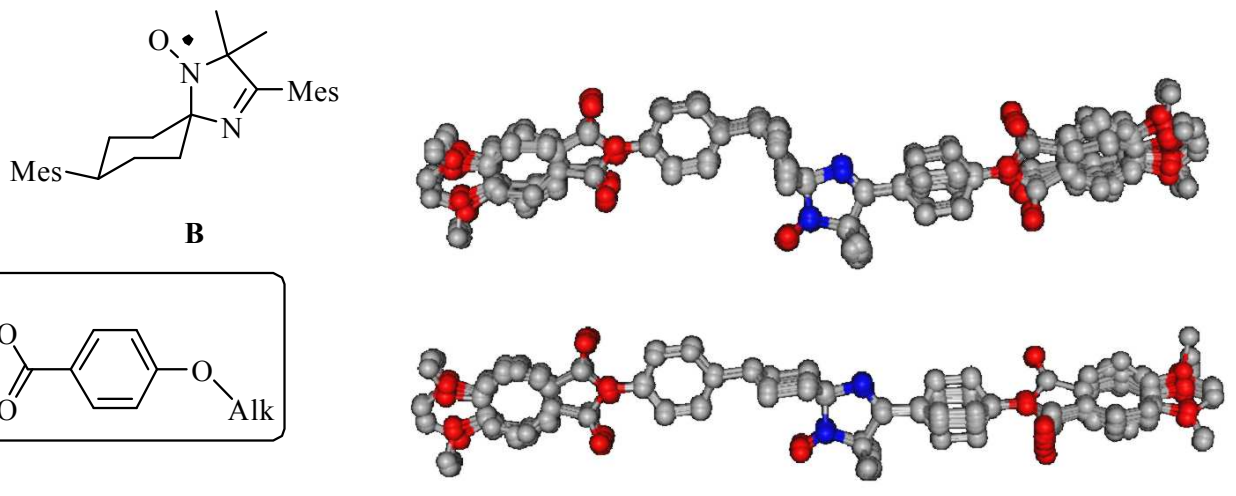

Figure 2 Superimposed conformers of diastereomers A (top) and $\mathbf{B}$ (bottom) $\left(\mathrm{Alk}=\mathrm{CH}_{3}\right)$.

Thus, we decided to prepare the target radicals using 1,4-bifunctional derivative of cyclohexane as the starting compound. An approach to the synthesis of functionally substituted spirocyclic radicals of 2,5-dihydroimidazole series includes condensation of the corresponding 2hydroxylamino ketones with cycloalkanones in the presence of ammonia followed by oxidation of intermediate 1-hydroxy-2,5-dihydroimidazoles ${ }^{32-35}$. Produced in such manner 3-imidazoline nitroxide radicals would be relatively inert toward many common chemical agents ${ }^{36}$ and could be modified on available functional groups without destruction of radical center. Condensation of commercially available 4-(4-hydroxyphenyl)cyclohexanone (7) with 2-hydroxylamino ketones 5 and $\mathbf{6}$ in methanol saturated with ammonia at ambient temperature gave imidazolines $\mathbf{8}$ and $\mathbf{9}$ with $70 \%$ and $97 \%$ yield, respectively. 


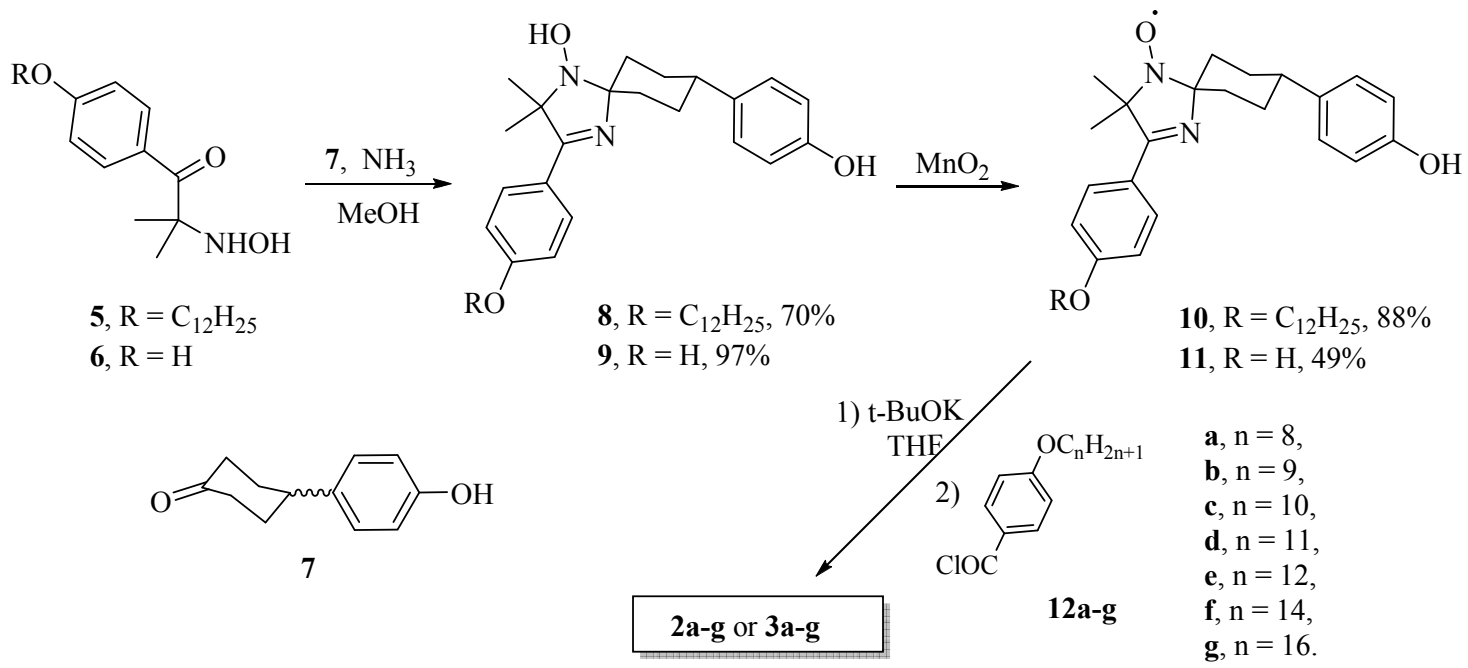

Scheme 1. Synthesis of nitroxides 2a-g and 3a-g.

The spatial molecular structure (3D-structure) depends on the conformation of "a central" cyclohexane fragment spirofused with an imidazoline ring due to the linearity of the substituents. Based on the chemical shift and coupling constants in the ${ }^{1} \mathrm{H}$ NMR spectrum of compound 9 , the proton $\mathrm{H} 8$ was assigned to the axial position and geminal to it 4-hydroxyphenyl substituent to equatorial. However, determination of configuration spirofused imidazoline cycle to the cyclohexane ring was a nontrivial task. To solve this problem, the NOESY and ROESY spectral information of 2,5-dihydroimidazole 9 was used together with the results of quantum chemical conformational analyses of its two possible stereoisomers. Consequently, 13 conformers were found for both isomers (excluding enantiomers originating from the chirality of twist and $\mathrm{sp}^{3}$ nitrogen atom) due to the conformation of the cyclohexane ring and the spatial arrangement of two aromatic rings ${ }^{37}$. These data suggest that the equatorial position of $\mathrm{NOH}$ fragment in 2,5dihydroimidazole as compared to the axial one has advantage by ca. $2 \mathrm{kcal} / \mathrm{mol}$ (according to DFT calculations). Comparison of spatial structures of low-energy conformers with ROESY data allowed us to identify $\mathbf{9}$ as the trans-ee-isomer (see Fig. 3). Similarly, compound $\mathbf{8}$ is assumed to take a trans-ee-isomer. An analogous substituent conformation was shown in a recent work by Serrano $e t a l^{38}$ for 4'-substituted cyclohexane-5-spirohydantoins which was consistent with that obtained by the X-ray crystallographic analysis. 


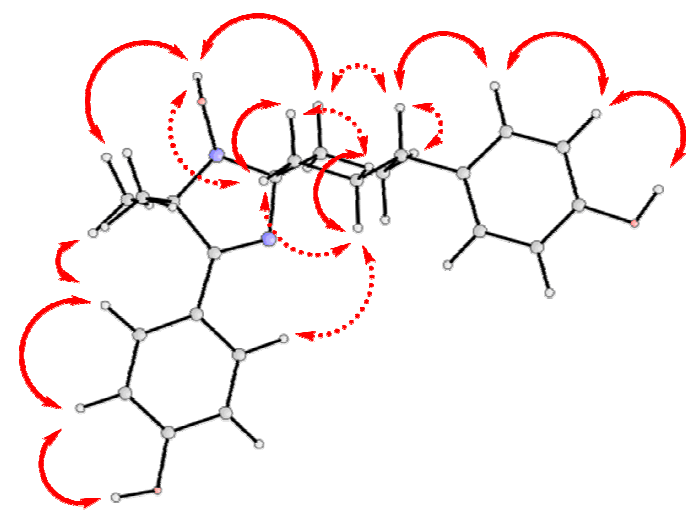

Figure 3. Optimized molecular structure of major trans-ee-conformer 9 by DFT/PBE/L1 (see Experimental) calculations. The red arrows indicate the ROESY cross-peaks; solid arrows indicate strong effects and dashed arrows - weak ones.

Thus, condensation of 2-hydroxylamino ketones with 4-substituted cyclohexanone in the presence of ammonia turned out to proceed with high stereoselectivity to furnish an A type isomer, apparently, more thermodynamically stable product.

Oxidation of imidazolines $\mathbf{8}$ and $\mathbf{9}$ with manganese dioxide in chloroform - methanol solution gave the corresponding radicals 10 and 11 with $88 \%$ and $49 \%$ yields, respectively. To perform acylation, phenoxide anions of nitroxides $\mathbf{1 0}$ and $\mathbf{1 1}$ were successfully generated with potassium tert-butoxide as a base. These anions were reacted with 4-alkoxybenzoic acid chlorides 12a-g to give the target nitroxides $\mathbf{2 a - g}$ and 3a-g.

For the synthesis of nitroxide 4 with two different mesogenic acyl groups $(n \neq m)$, radical 11 was initially treated with one equiv of tert-BuOK and then one equiv of 4-octyloxybenzoic acid chloride (12a) was added to the reaction mixture to give monoacyl derivative $\mathbf{1 3}$ in $28 \%$ yield after the starting radical 11 were separated chromatographically. To characterize the product $\mathbf{1 3}$, it was reduced by $\mathrm{Zn} / \mathrm{NH}_{4} \mathrm{Cl}$ to give the hydroxylamine 14. Comparison of $\mathrm{NMR}{ }^{1} \mathrm{H}$ and ${ }^{13} \mathrm{C}$ spectra of 14 with 9 verified the monoacylation at the more acidic phenolic group (Scheme 2). Reaction of nitroxide $\mathbf{1 3}$ with 4-nonyloxybenzoic acid chloride (12b) afforded the target radical 4 possessing two different mesogenic acyl groups in $21 \%$ yield. 

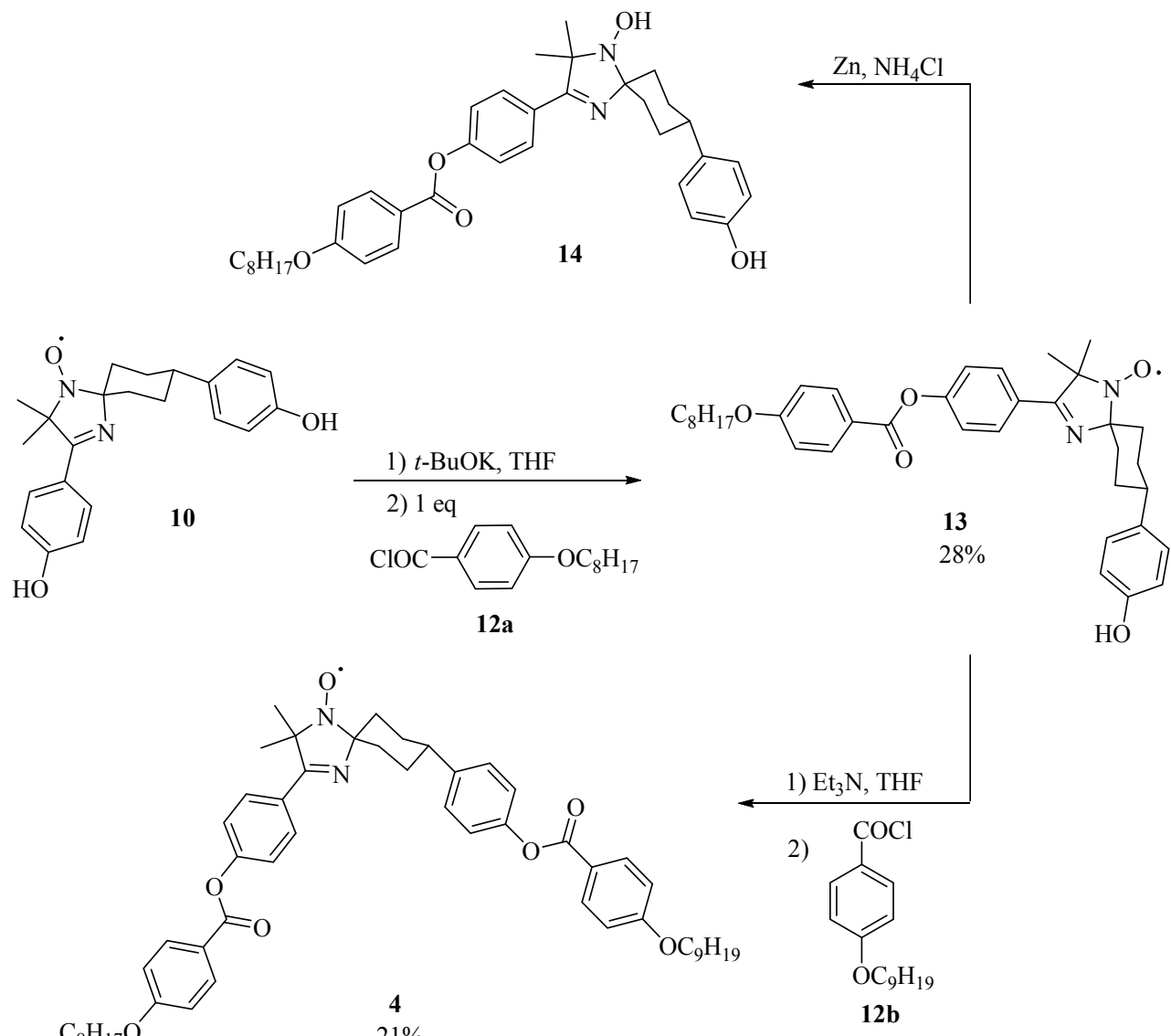

Scheme 2. Synthesis of nitroxide 4 with two different mesogenic acyl groups.

We performed DSC analysis for nitroxides $\mathbf{2 a - g}$ and 3a-g in the temperature range from 25 ${ }^{\circ} \mathrm{C}$ to $120{ }^{\circ} \mathrm{C}$ under nitrogen gas. All radicals are stable in this temperature range. For nitroxides $\mathbf{2 c}, \mathbf{d}, \mathbf{g}(\mathrm{n}=10,11,16)$ and $\mathbf{3 a}, \mathbf{c - f}(\mathrm{n}=8,10,11,14,12)$, there are only melting peaks in the heating run. For radicals $\mathbf{2 a}, \mathbf{b}, \mathbf{e}, \mathbf{f}(\mathrm{n}=8,9,12,14)$ and $\mathbf{3 b}, \mathbf{g}(\mathrm{n}=9,16)$, there are series of peaks in the heating and cooling runs (see Experimental). Observation of phase transitions for compounds $\mathbf{2 a}, \mathbf{b}, \mathbf{e}, \mathbf{f}$ and $\mathbf{3 b}, \mathbf{g}$ by using polarizing optical microscope showed that registered sequence of peaks correspond to polymorphous transitions, rather than the generation of mesophases in this temperature region. As a result, synthesized paramagnetic compounds did not reveal LC behavior. Apparently, this may be connected with unfavorable molecular shapes of spirocyclic nitroxides whose Z-shaped backbones were formed at the initial stage.

\section{Conclusions}

We have developed a convenient approach to the synthesis of stable nitroxides of 2,5dihydroimidazole series bearing two identical and two different mesogenic groups located in 
both sides of spirofused heterocyclic and cyclohexane frameworks. On the basis of NMR studies, trans-ee configuration of substituents in 1,4-cyclohexane ring was established. As a consequence, none of the obtained nitroxides demonstrated mesomorphous behavior due to nonlinearity of molecules.

\section{Experimental Section}

General. Analytical and spectroscopic studies were performed in the Chemical Service Center of collective use of the SB RAS. Melting points and DSC-thermograms were obtained by FP $81 \mathrm{HT}$ «METTLER TOLEDO» device. IR spectra were recorded on a Vector-22 Bruker spectrometer in $\mathrm{KBr} .{ }^{1} \mathrm{H}$ and ${ }^{13} \mathrm{C}$ NMR spectra were recorded on Bruker AV-300, AV-400 and AV-600 spectrometers for solutions of compounds in $\mathrm{CDCl}_{3}+\mathrm{CD}_{3} \mathrm{OD}, \mathrm{CD}_{3} \mathrm{OD}$, DMSO- $\mathrm{d}_{6}$, chemical shifts were recorded in parts per million $(\mathrm{ppm})$ relative to internal standard residual solvent signals $-\mathrm{CDCl}_{3}\left(\delta_{\mathrm{H}} 7.24 \mathrm{ppm}, \delta_{\mathrm{C}} 77.36 \mathrm{ppm}\right), \mathrm{CD}_{3} \mathrm{OD}\left(\delta_{\mathrm{H}} 3.34 \mathrm{ppm}, \delta_{\mathrm{C}} 49.00 \mathrm{ppm}\right)$, DMSO-d 6 $\left(\delta_{\mathrm{H}} 2.50 \mathrm{ppm}, \delta_{\mathrm{C}} 39.50 \mathrm{ppm}\right)$. Structures of compounds were established by conventional oneand two-dimensional NMR spectra. Mixed time $0.2 \mathrm{~s}$ for NOESY and ROESY of compound $\mathbf{8}$ was used. ESR spectra of radicals were recorded on a Bruker 300 spectrometer for $10^{-4} \mathrm{M}$ chloroform solutions. Reactions were monitored by TLC on silica gel with Sorbfil UV-254 plates. The silica gel utilized for column chromatography (CC) was purchased from ACROS, 0.060-0.200 $\mathrm{mm}$. All chemicals and solvents were commercial reagent quality and used without further purification unless otherwise stated. Dry tetrahydrofuran (THF) was obtained by distillation over $\mathrm{LiH} / \mathrm{CaH}_{2}$ mixture. 4-(4-Hydroxyphenyl)cyclohexanone was purchased from Aldrich. 2-Hydroxylamino ketones 5, 6 were prepared by analogy with patent ${ }^{39}$. 4-Alkoxyphenyl benzoic acids ${ }^{40}$ were obtained by literature procedure.

The initial set of conformers for compounds A, B and $\mathbf{9}$ was obtained by using ChemAxon's Marvin (conformers plugin) ${ }^{41}$, Verachem Vconf ${ }^{42}$ and Confab $^{43}$, then structures were optimized by $\mathrm{RM}^{44}$ with the MOPAC2012 program $^{45}$, and for 9 by the density functional theory (functional $\mathrm{PBE}^{46}$, basis $\mathrm{L} 1\left(\Lambda 01^{47}\right.$, cc-pVDZ analog), with the PRIRODA program ${ }^{47}$ ). The chemical shifts were calculated by GIAO/DFT/PBE (basis L22 (L22, cc-pCVTZ analog), with the PRIRODA program. For quantum chemical calculations, we used the cluster of the Information Computation Center, Novosibirsk State University ${ }^{48}$. All results of DFT calculations are available ${ }^{37}$.

\section{2,2-Dimethyl-3-(4-dodecyloxy)phenyl)-1-hydroxy-8-(4-hydroxyphenyl)-1,4-diazaspiro[4,5]-} deca-3-en (8). The mixture of 4-(4-hydroxyphenyl)cyclohexanone (7) $(0.570 \mathrm{~g}, 3 \mathrm{mmol})$ and 1(4-dodecyloxyphenyl)-2-(hydroxylamino)-2-methylpropan-1-one hydrochloride (5) (1.200 g, 3 $\mathrm{mmol})$ in $\mathrm{MeOH}(30 \mathrm{ml})$ saturated with ammonia stirred at $\mathrm{rt}$ during $4 \mathrm{~h}$. Precipitate formed was filtrated, washed by water and $\mathrm{MeOH}$. Analytical sample was purified by crystallization from mixture $\mathrm{CHCl}_{3}-\mathrm{MeOH}$. White powder, yield $70 \%, 1.13 \mathrm{~g}, \mathrm{mp} 145-146{ }^{\circ} \mathrm{C}\left(\mathrm{CHCl}_{3}-\mathrm{MeOH}\right)$; IR 
$\left(v_{\max }, \mathrm{cm}^{-1}\right): 3228(\mathrm{OH}), 1602,1573(\mathrm{C}=\mathrm{N}) .{ }^{1} \mathrm{H}$ NMR $\left(400 \mathrm{MHz}, \mathrm{DMSO}-d_{6}\right): \delta_{\mathrm{H}} 0.83(3 \mathrm{H}, \mathrm{m}$, $\left.\mathrm{CH}_{2} \underline{\mathrm{C}}_{3}\right), 1.20\left(16 \mathrm{H}, \mathrm{m}, 8 \mathrm{C}_{2}\right), 1.34\left(6 \mathrm{H}, \mathrm{s}, \mathrm{Me}-11\right.$ and $\left.\mathrm{Me}-12+2 \mathrm{H}, \mathrm{m}, \mathrm{C}_{2}\right), 1.49(2 \mathrm{H}, \mathrm{m}, \mathrm{H}-$ $6 e, 10 e), 1.68\left(2 \mathrm{H}, \mathrm{m}, \mathrm{H}-7 e, 9 e+2 \mathrm{H}, \mathrm{m}, \mathrm{OCH}_{2} \underline{\mathrm{C}}_{2}\right),[1.86-2.04](4 \mathrm{H}, \mathrm{m}, \mathrm{H}-6 a, 7 a, 9 a, 10 a)$, $2.45(1 \mathrm{H}, \mathrm{m}, \mathrm{H}-8 a), 3.96\left(2 \mathrm{H}, \mathrm{m}, \mathrm{OC}_{2}\right), 6.65\left(2 \mathrm{H}, \mathrm{d},{ }^{3} J_{3^{\prime \prime}, 2^{\prime \prime}} 8.2 \mathrm{~Hz}, \mathrm{H}-3^{\prime \prime}, 5^{\prime \prime}\right), 6.93\left(2 \mathrm{H}, \mathrm{d},{ }^{3} J_{3^{\prime}, 2^{\prime}}\right.$ $\left.8.4 \mathrm{~Hz}, \mathrm{H}-3^{\prime}, 5^{\prime}\right), 7.00$ (2H, d, $\left.{ }^{3} J_{2^{\prime \prime}, 3^{\prime \prime}} 8.2 \mathrm{~Hz}, \mathrm{H}-2^{\prime \prime}, 6^{\prime \prime}\right), 7.56$ (1H, s, NOH) 7.78 (2H, d, arom., $\left.{ }^{3} J_{2^{\prime}, 3^{\prime}} 8.4 \mathrm{~Hz}, \mathrm{H}-2^{\prime}, 6^{\prime}\right), 9.06(1 \mathrm{H}, \mathrm{s}, \mathrm{OH}) .{ }^{13} \mathrm{C}$ NMR $\left(100 \mathrm{MHz}, \mathrm{DMSO}-d_{6}\right): \delta_{\mathrm{C}} 24.58(\mathrm{C}-11, \mathrm{C}-12)$, 25.46 (C-7,C-9), 31.30 (C-6, C-10), 42.41 (C-8), 67.54 (C-2), 88.48 (C-5), 114.22 (C-3',C-5'), 114.97 (C-3",C-5"), 125.55 (C-1'), 127.38 (C-2",C-6"), 129.23 (C-2',C-6'), 137.42 (C-1"), 155.31 (C-4"), 160.14 (C-4), 171.54 (C-3). Alkyl chain signals: $13.95\left(\underline{\mathrm{CH}}_{3}\right), 22.10,28.57,28.72,28.97$, 29.02, 31.51, 35.82, $69.37\left(\mathrm{OCH}_{2}\right)$. Anal. Calcd for $\mathrm{C}_{34} \mathrm{H}_{50} \mathrm{~N}_{2} \mathrm{O}_{3}$ (534.77): C, 76.36; H, 9.42; N, 5.24\%. Found: C, 76.22; H, 9.35; N, 5.26\%.

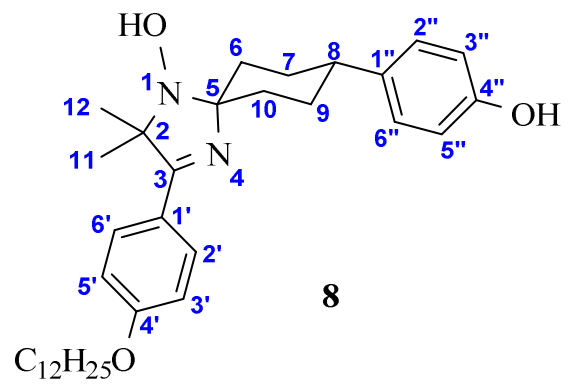

2,2-Dimethyl-3-(4-dodecyloxy)phenyl)-8-(4-hydroxyphenyl)-1,4-diazaspiro[4,5]deca-3-en 1oxyl (10). A suspension of hydroxylamine 8 (1.000 g, $1.87 \mathrm{mmol})$ and $\mathrm{MnO}_{2}(1.290 \mathrm{~g}, 15 \mathrm{mmol})$ in the mixture $\mathrm{CHCl}_{3}-\mathrm{MeOH}, 3: 1(30 \mathrm{ml})$ was stirred during $2 \mathrm{~h}$ at rt. Excess of the oxidant was filtrated and the solvent was evaporated. Solid residue was purified by hot crystallization from ethanol. Yellow powder, yield 88\%, $0.879 \mathrm{~g}, \mathrm{mp} 140-142{ }^{\circ} \mathrm{C}(\mathrm{EtOH})$; IR $\left(v_{\max }, \mathrm{cm}^{-1}\right): 3410$ $(\mathrm{OH}), 1608,1589(\mathrm{C}=\mathrm{N})$. ESR: $\mathrm{t}, \mathrm{A}_{\mathrm{N}} 1.445 \mathrm{mT}$, giso 2.0058. Anal. Calcd for $\mathrm{C}_{34} \mathrm{H}_{49} \mathrm{~N}_{2} \mathrm{O}_{3}$ (533.76): C, 76.51; H, 9.25; N, 5.25\%. Found: C, 76.40; H, 9.15; N, 5.26\%.

\section{8-[4-(4-(Alkoxy)benzoyloxy)phenyl]-2,2-dimethyl-3-(4-dodecyloxyphenyl)-1,4-diazaspiro-}

[4,5]deca-3-en 1-oxyls 2a-g (general procedure). A solution of 4-alkyloxybenzoic acid (1.8 $\mathrm{mmol}$ ) in thionyl chloride $\left(2 \mathrm{ml}\right.$ ) was refluxed during $1.5 \mathrm{~h}$. The excess of $\mathrm{SOCl}_{2}$ was removed in vacuum and residue was diluted with dry THF $(10 \mathrm{ml})$, after that solution was added upon stirring to suspension of nitroxide $\mathbf{1 0}(0.160 \mathrm{~g}, 0.3 \mathrm{mmol})$ and potassium tert-butoxide $(0.201 \mathrm{~g}$, $1.8 \mathrm{mmol})$ in dry THF $(10 \mathrm{ml})$. The mixture was stirred at $\mathrm{rt}$ during $1 \mathrm{~h}$ and concentrated. Residue was treated with diethyl ether $(15 \mathrm{ml})$, washed by saturated aq solution of $\mathrm{NaHCO}_{3}$ $(2 \times 10 \mathrm{ml})$ and water $(2 \times 10 \mathrm{ml})$. The organic layer was separated, dried over $\mathrm{MgSO}_{4}$ and the solvent evaporated. Residue was purified by CC (hexane-EtOAc, 4:1). Analytical sample was prepared by crystallization from ethanol.

2,2-Dimethyl-3-(4-dodecyloxyphenyl)-8-[4-(4-(octyloxy)benzoyloxy)phenyl]-1,4-diazaspiro[4,5]deca-3-en 1-oxyl (2a). Yellow powder, yield 59\%, $0.136 \mathrm{~g}$. There are two peaks at the DSC curve on the heating run: $100{ }^{\circ} \mathrm{C}$ and $112^{\circ} \mathrm{C}$. IR $\left(v_{\max }, \mathrm{cm}^{-1}\right): 1726(\mathrm{C}=\mathrm{O}), 1606,1566(\mathrm{C}=\mathrm{N})$. 
ESR: t, $\mathrm{A}_{\mathrm{N}} 1.455 \mathrm{mT}, \mathrm{g}_{\text {iso }}$ 2.0058. Anal. Calcd for $\mathrm{C}_{49} \mathrm{H}_{69} \mathrm{~N}_{2} \mathrm{O}_{5}$ (766.08): C, 76.82; $\mathrm{H}, 9.08 ; \mathrm{N}$, 3.66\%. Found: C, 77.01; H, 8.99; N, 3.79\%.

2,2-Dimethyl-3-(4-dodecyloxyphenyl)-8-[4-(4-(nonyloxy)benzoyloxy)phenyl]-1,4-diazaspiro[4,5]deca-3-en 1-oxyl (2b). Yellow powder, yield 42\%, $0.098 \mathrm{~g}$. There are two peaks at the DSC curve on the heating run: $99{ }^{\circ} \mathrm{C}$ and $106{ }^{\circ} \mathrm{C}$. IR $\left(v_{\max }, \mathrm{cm}^{-1}\right): 1724(\mathrm{C}=\mathrm{O}), 1606,1566(\mathrm{C}=\mathrm{N})$. ESR: $t, \mathrm{~A}_{\mathrm{N}} 1.455 \mathrm{mT}$, giso 2.0058. Anal. Calcd for $\mathrm{C}_{50} \mathrm{H}_{71} \mathrm{~N}_{2} \mathrm{O}_{5}$ (780.11): C, 76.98; H, 9.17; N, 3.59. Found: C, 76.93; H, 9.09; N, 3.63.

8-[4-(4-(Decyloxy)benzoyloxy)phenyl]-2,2-dimethyl-3-(4-dodecyloxyphenyl)-1,4-diazaspiro[4,5]deca-3-en 1-oxyl (2c). Yellow powder, yield 53\%, $0.126 \mathrm{~g}, \mathrm{mp} \mathrm{103-105}{ }^{\circ} \mathrm{C}(\mathrm{EtOH})$; IR $\left(v_{\max }, \mathrm{cm}^{-1}\right): 1722(\mathrm{C}=\mathrm{O}), 1604,1566(\mathrm{C}=\mathrm{N})$. ESR: $\mathrm{t}, \mathrm{A}_{\mathrm{N}} 1.455 \mathrm{mT}, \mathrm{g}_{\text {iso }}$ 2.0058. Anal. Calcd for $\mathrm{C}_{51} \mathrm{H}_{73} \mathrm{~N}_{2} \mathrm{O}_{5}$ (794.14): C, 77.13; H, 9.27; N, 3.53\%. Found: C, 76.95; H, 9.17; N, 3.48\%.

2,2-Dimethyl-3-(4-dodecyloxyphenyl)-8-[4-(4-(undecyloxy)benzoyloxy)phenyl]-1,4diazaspiro[4,5]deca-3-en 1-oxyl (2d). Yellow powder, yield 79\%, 0.191 g, mp 93-95 ${ }^{\circ} \mathrm{C}$ $(\mathrm{EtOH})$; IR $\left(v_{\max }, \mathrm{cm}^{-1}\right): 1722(\mathrm{C}=\mathrm{O}), 1606,1581(\mathrm{C}=\mathrm{N})$. ESR: $\mathrm{t}, \mathrm{A}_{\mathrm{N}} 1.455 \mathrm{mT}, \mathrm{g}_{\text {iso }}$ 2.0058. Anal. Calcd for $\mathrm{C}_{52} \mathrm{H}_{75} \mathrm{~N}_{2} \mathrm{O}_{5}$ (808.16): C, 77.28; H, 9.35; N, 3.47\%. Found: C, 77.19; H, 9.33; N, $3.40 \%$.

2,2-Dimethyl-8-[4-(4-(dodecyloxy)benzoyloxy)phenyl]-3-(4-dodecyloxyphenyl)-1,4diazaspiro[4,5]deca-3-en 1-oxyl (2e). Yellow powder,yield 49\%, $0.120 \mathrm{~g}$. There are two peaks at the DSC curve on the heating run: $93{ }^{\circ} \mathrm{C}$ and $109^{\circ} \mathrm{C}$. IR $\left(v_{\max }, \mathrm{cm}^{-1}\right): 1722(\mathrm{C}=\mathrm{O}), 1604,1566$ $(\mathrm{C}=\mathrm{N})$. ESR: $\mathrm{t}, \mathrm{A}_{\mathrm{N}} 1.455 \mathrm{mT}$, giso 2.0058. Anal. Calcd for $\mathrm{C}_{53} \mathrm{H}_{77} \mathrm{~N}_{2} \mathrm{O}_{5}(822.19): \mathrm{C}, 77.42 ; \mathrm{H}$, 9.44; N, 3.41\%. Found: C, 77.34; H, 9.39; N, 3.43\%.

2,2-Dimethyl-3-(4-dodecyloxyphenyl)-8-[4-(4-(tetradecyloxy)benzoyloxy)phenyl]-1,4diazaspiro[4,5]deca-3-en 1-oxyl (2f). Yellow powder, yield 86\%, $0.219 \mathrm{~g}$. There are tree peaks at the DSC curve on the heating run: $55^{\circ} \mathrm{C}, 62{ }^{\circ} \mathrm{C}$ and $94{ }^{\circ} \mathrm{C}$ and tree peaks on the cooling run: $69{ }^{\circ} \mathrm{C}, 57^{\circ} \mathrm{C}$ and $52{ }^{\circ} \mathrm{C}$. IR $\left(v_{\max }, \mathrm{cm}^{-1}\right): 1722(\mathrm{C}=\mathrm{O}), 1606,1581(\mathrm{C}=\mathrm{N})$. ESR: $\mathrm{t}, \mathrm{A}_{\mathrm{N}} 1.455 \mathrm{mT}$, $\mathrm{g}_{\text {iso }}$ 2.0058. Anal. Calcd for $\mathrm{C}_{55} \mathrm{H}_{81} \mathrm{~N}_{2} \mathrm{O}_{5}$ (850.24): C, 77.69; H, 9.60; N, 3.29\%. Found: C, 77.81; H, 9.68; N, 3.20\%.

2,2-Dimethyl-3-(4-dodecyloxyphenyl)-8-[4-(4-(hexadecyloxy)benzoyloxy)phenyl]-1,4diazaspiro[4,5]deca-3-en 1-oxyl (2g). Yellow powder, yield 49\%, 0.129 g, mp 109-111 ${ }^{\circ} \mathrm{C}$ $(\mathrm{EtOH})$; IR $\left(v_{\max }, \mathrm{cm}^{-1}\right): 1722(\mathrm{C}=\mathrm{O}), 1606,1581(\mathrm{C}=\mathrm{N})$. ESR: t, $\mathrm{A}_{\mathrm{N}} 1.455 \mathrm{mT}, \mathrm{g}_{\text {iso }} 2.0058$. Anal. Calcd for $\mathrm{C}_{57} \mathrm{H}_{85} \mathrm{~N}_{2} \mathrm{O}_{5}$ (878.30): C, 77.95; H, 9.75; N, 3.19\%. Found: C, 77.90; H, 9.64; N, $3.11 \%$.

3,8-Bis(4-hydroxyphenyl)-2,2-dimethyl-1-hydroxy-1,4-diazaspiro[4,5]deca-3-en (9). A solution of 2-(hydroxylamino)-1-(4-hydroxyphenyl)-2-methylpropan-1-one (6) (0.591 g, 3 $\mathrm{mmol}$ ) and 4-(4-hydroxyphenyl)cyclohexanone (7) $(0.570 \mathrm{~g}, 3 \mathrm{mmol})$ in $30 \mathrm{ml} \mathrm{MeOH}$ saturated with ammonia was kept at $\mathrm{rt}$ during $4 \mathrm{~h}$. Solvent was removed under reduced pressure, water (15 $\mathrm{ml}$ ) was added, precipitate formed was filtrated, washed with water and dried on air. White powder, yield 97\%, $1.07 \mathrm{~g}, \mathrm{mp} 165-167{ }^{\circ} \mathrm{C}\left(\mathrm{H}_{2} \mathrm{O}\right)$; IR $\left(v_{\max }, \mathrm{cm}^{-1}\right): 3425,3247(\mathrm{OH}), 1610$ $(\mathrm{C}=\mathrm{N}) .{ }^{1} \mathrm{H}$ NMR $\left(600 \mathrm{MHz}, \mathrm{DMSO}-d_{6}\right): \delta_{\mathrm{H}} 1.37\left(6 \mathrm{H}, \mathrm{s}, \mathrm{Me}-11\right.$ and Me-12), $1.50\left(2 \mathrm{H}, \mathrm{br} . \mathrm{d},{ }^{2} J\right.$ $12.4 \mathrm{~Hz}, \mathrm{H}-6 e, 10 e), 1.71\left(2 \mathrm{H}\right.$, br.d, $\left.{ }^{2} J 11.5 \mathrm{~Hz}, \mathrm{H}-7 e, 9 e\right), 1.91\left(2 \mathrm{H}\right.$, br.ddd, ${ }^{3} J_{7 a, 6 a} 13.2 \mathrm{~Hz},{ }^{3} J_{7 a}$, 
$\left.{ }_{8 a} 12.2 \mathrm{~Hz},{ }^{2} J 11.5 \mathrm{~Hz}, \mathrm{H}-7 a, 9 a\right), 2.02\left(2 \mathrm{H}, \mathrm{br} . \mathrm{dd},{ }^{3} J_{6 a, 7 a} 13.2 \mathrm{~Hz},{ }^{2} J 12.4 \mathrm{~Hz}, \mathrm{H}-6 a, 10 a\right), 2.47$ (1H, br.dd, $\left.{ }^{3} J_{8 a, 7 a}=J_{8 a, 9 a} 12.2 \mathrm{~Hz}, \mathrm{H}-8 a\right), 6.68\left(2 \mathrm{H}, \mathrm{d},{ }^{3} J_{3^{\prime \prime}, 2^{\prime \prime}} 8.1 \mathrm{~Hz}, \mathrm{H}-3^{\prime \prime}, 5^{\prime \prime}\right), 6.82\left(2 \mathrm{H}, \mathrm{d},{ }^{3} J_{3^{\prime}, 2^{\prime}}\right.$ $\left.8.3 \mathrm{~Hz}, \mathrm{H}-3^{\prime}, 5^{\prime}\right), 7.04\left(2 \mathrm{H}, \mathrm{d},{ }^{3} J_{2^{\prime \prime}}, 3^{\prime \prime} 8.1 \mathrm{~Hz}, \mathrm{H}-2^{\prime \prime}, 6^{\prime \prime}\right), 7.58$ (1H, s, NOH), $7.74\left(2 \mathrm{H}, \mathrm{d},{ }^{3} J_{2^{\prime}, 3^{\prime}} 8.3\right.$ $\left.\mathrm{Hz}, \mathrm{H}-2^{\prime}\right), 9.10\left(1 \mathrm{H}, \mathrm{s}, \mathrm{OH}-4^{\prime \prime}\right), 9.87\left(1 \mathrm{H}, \mathrm{s}, \mathrm{OH}-4^{\prime}\right) .{ }^{13} \mathrm{C}$ NMR (150 MHz, DMSO- $\left.d_{6}\right): \delta_{\mathrm{C}} 24.58 \mathrm{q}$ (Me-11, 12), 31.45 t (C-7, 9), 35.78 t (C-6, 10), 42.34 d (C-8), 69.25 s (C-2), 88.25 s (C-5), $114.91 \mathrm{~d}\left(\mathrm{C}-3^{\prime \prime}, 5^{\prime \prime}\right), 115.08 \mathrm{~d}\left(\mathrm{C}-3^{\prime}, 5^{\prime}\right), 124.09 \mathrm{~s}\left(\mathrm{C}-1^{\prime}\right), 127.34 \mathrm{~d}\left(\mathrm{C}-2^{\prime \prime}, 6^{\prime \prime}\right), 129.29 \mathrm{~d}\left(\mathrm{C}-2^{\prime}, 6^{\prime}\right)$, $137.39 \mathrm{~s}\left(\mathrm{C}-1^{\prime \prime}\right), 155.24 \mathrm{~s}\left(\mathrm{C}-4^{\prime \prime}\right), 159.14 \mathrm{~s}\left(\mathrm{C}-4^{\prime}\right), 171.53 \mathrm{~s}(\mathrm{C}-3)$. Anal. Calcd for $\mathrm{C}_{22} \mathrm{H}_{26} \mathrm{~N}_{2} \mathrm{O}_{3}$ (366.45): C, 72.11; H, 7.15; N, 7.64\%. Found: C, 72.01; H, 7.13; N, 7.58\%.

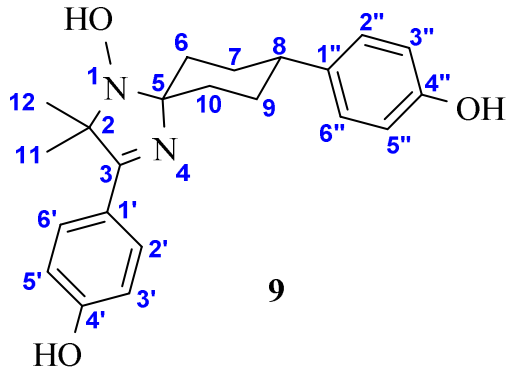

3,8-Bis(4-hydroxyphenyl)-2,2-dimethyl-1,4-diazaspiro[4,5]deca-3-en 1-oxyl (11). A suspension of hydroxylamine $9(0.96 \mathrm{~g}, 2.62 \mathrm{mmol})$ and manganese dioxide $(1.13 \mathrm{~g}, 13.15$ mmol) in the mixture of $20 \mathrm{ml} \mathrm{MeOH}$ and $5 \mathrm{ml} \mathrm{CHCl}_{3}$ was stirred at ambient temperature during $2 \mathrm{~h}$. The excess of oxidant was filtrated and the solvent evaporated under reduced pressure. Residue was purified by CC (hexane-EtOAc, 1:1). Analytical sample of radical 11 was obtained according next procedure: a solution of $30 \mathrm{mg}$ crude nitroxide in $3 \%$ aq sodium hydroxide was acidified carefully by $5 \%$ aq $\mathrm{HCl}$ until $\mathrm{pH} \sim 6$, precipitate was filtrated, washed by water and dried on air. Yellow crystals, yield $49 \%, 0.47 \mathrm{~g}, \mathrm{mp} 156-157{ }^{\circ} \mathrm{C}\left(\mathrm{H}_{2} \mathrm{O}\right)$; IR $\left(v_{\max }, \mathrm{cm}^{-1}\right): 3228(\mathrm{OH})$, 1614, $1595(\mathrm{C}=\mathrm{N})$. ESR: t, $\mathrm{A}_{\mathrm{N}} 1.412 \mathrm{mT}$, giso 2.0058. Anal. Calcd for $\mathrm{C}_{22} \mathrm{H}_{25} \mathrm{~N}_{2} \mathrm{O}_{3}$ (365.45): $\mathrm{C}$, $72.31 ; \mathrm{H}, 6.90 ; \mathrm{N}, 7.67 \%$. Found: C, 72.48; H, 6.83; N, 7.61\%.

3,8-Bis[4-(4-alkoxy)benzoyloxy)phenyl]-2,2-dimethyl-1,4-diazaspiro[4,5]deca-3-en 1-oxyls 3a-g were prepared according to the synthetic procedure for compounds $\mathbf{2 a - g}$ from nitroxide $\mathbf{1 1}$ and 3 equiv of 4-alkoxybenzoic acid chlorides 12a-g.

3,8-Bis[4-(4-octyloxy)benzoyloxy)phenyl]-2,2-dimethyl-1,4-diazaspiro[4,5]deca-3-en 1-oxyl (3a). Yellow powder, yield 25\%, $0.063 \mathrm{~g}, \mathrm{mp} 127-129^{\circ} \mathrm{C}(\mathrm{EtOH}) ; \mathrm{IR}\left(v_{\max }, \mathrm{cm}^{-1}\right): 1730(\mathrm{C}=\mathrm{O})$, 1604, $1571(\mathrm{C}=\mathrm{N})$. ESR: $\mathrm{t}, \mathrm{A}_{\mathrm{N}} 1.455 \mathrm{mT}$, $\mathrm{g}_{\text {iso }}$ 2.0058. Anal. Calcd for $\mathrm{C}_{52} \mathrm{H}_{65} \mathrm{~N}_{2} \mathrm{O}_{7}$. (830.08): $\mathrm{C}$, 75.24; H, 7.89; N, 3.37\%. Found: C, 75.36; H, 7.89; N, 3.25\%.

3,8-Bis[4-(4-nonyloxy)benzoyloxy)phenyl]-2,2-dimethyl-1,4-diazaspiro[4,5]deca-3-en 1-oxyl (3b). Yellow powder, yield $47 \%, 0.121 \mathrm{~g}$. There are two peaks at the DSC curve on the heating run: $100{ }^{\circ} \mathrm{C}$ and $112{ }^{\circ} \mathrm{C}$ and two peaks on the cooling run: $101{ }^{\circ} \mathrm{C}, 88^{\circ} \mathrm{C}$. IR $\left(v_{\max }, \mathrm{cm}^{-1}\right): 1732$ $(\mathrm{C}=\mathrm{O}), 1606,1577(\mathrm{C}=\mathrm{N})$. ESR: $\mathrm{t}, \mathrm{A}_{\mathrm{N}} 1.455 \mathrm{mT}$, giso 2.0058. Anal. Calcd for $\mathrm{C}_{54} \mathrm{H}_{69} \mathrm{~N}_{2} \mathrm{O}_{7}$ (858.13): C, 75.58; H, 8.10; N, 3.26\%. Found: C, 75.70; H, 8.11; N, 3.20\%. 
3,8-Bis[4-(4-decyloxy)benzoyloxy)phenyl]-2,2-dimethyl-1,4-diazaspiro[4,5]deca-3-en 1-oxyl (3c). Yellow powder, yield 30\%, 0.080 g, mp 110-112 ${ }^{\circ} \mathrm{C}(\mathrm{EtOH}) ; \mathrm{IR}\left(v_{\max }, \mathrm{cm}^{-1}\right): 1727(\mathrm{C}=\mathrm{O})$, 1604, $1579(\mathrm{C}=\mathrm{N})$. ESR: $\mathrm{t}, \mathrm{A}_{\mathrm{N}} 1.455 \mathrm{mT}$, $\mathrm{g}_{\text {iso }}$ 2.0058. Anal. Calcd for $\mathrm{C}_{56} \mathrm{H}_{73} \mathrm{~N}_{2} \mathrm{O}_{7}$.(886.19): $\mathrm{C}$, $75.90 ; \mathrm{H}, 8.30 ; \mathrm{N}, 3.16 \%$. Found: C, 76.10; H, 8.25; N, 3.13\%.

3,8-Bis[4-(4-undecyloxy)benzoyloxy)phenyl]-2,2-dimethyl-1,4-diazaspiro[4,5]deca-3-en 1-

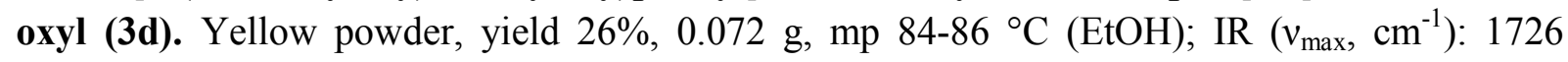
$(\mathrm{C}=\mathrm{O}), 1606,1579(\mathrm{C}=\mathrm{N})$. ESR: t, $\mathrm{A}_{\mathrm{N}} 1.455 \mathrm{mT}, \mathrm{g}_{\text {iso }}$ 2.0058. Anal. Calcd for $\mathrm{C}_{58} \mathrm{H}_{77} \mathrm{~N}_{2} \mathrm{O}_{7}$ (914.24): C, 76.20; H, 8.49; N, 3.06\%. Found: C, 76.30; H, 8.56; N, 3.05\%.

3,8-Bis[4-(4-dodecyloxy)benzoyloxy)phenyl]-2,2-dimethyl-1,4-diazaspiro[4,5]deca-3-en 1oxyl (3e). Yellow powder, yield 52\%, 0.147 g, mp 106-108 ${ }^{\circ} \mathrm{C}(\mathrm{EtOH}) ; \mathrm{IR}\left(\mathrm{v}_{\max }, \mathrm{cm}^{-1}\right): 1726$ $(\mathrm{C}=\mathrm{O}), 1606,1579(\mathrm{C}=\mathrm{N})$. ESR: t, $\mathrm{A}_{\mathrm{N}} 1.455 \mathrm{mT}$, giso 2.0058. Anal. Calcd for $\mathrm{C}_{60} \mathrm{H}_{81} \mathrm{~N}_{2} \mathrm{O}_{7}$ (942.29): C, 76.48; H, 8.66; N, 2.97\%. Found: C, 76.28; H, 8.67; N, 2.90\%.

3,8-Bis[4-(4-tetradecyloxy)benzoyloxy)phenyl]-2,2-dimethyl-1,4-diazaspiro[4,5]deca-3-en 1oxyl (3f). Yellow powder, yield $35 \%, 0.105 \mathrm{~g}, \mathrm{mp} 92-94{ }^{\circ} \mathrm{C}(\mathrm{EtOH})$; IR $\left(v_{\max }, \mathrm{cm}^{-1}\right): 1730$ $(\mathrm{C}=\mathrm{O}), 1606,1579(\mathrm{C}=\mathrm{N})$. ESR: t, $\mathrm{A}_{\mathrm{N}} 1.455 \mathrm{mT}, \mathrm{g}_{\text {iso }}$ 2.0058. Anal. Calcd for $\mathrm{C}_{64} \mathrm{H}_{89} \mathrm{~N}_{2} \mathrm{O}_{7}$. (998.40): C, 76.99; H, 8.99; N, 2.81\%. Found: C, 77.03; H, 9.03; N, 2.77\%.

3,8-Bis[4-(4-hexadecyloxy)benzoyloxy)phenyl]-2,2-dimethyl-1,4-diazaspiro[4,5]deca-3-en 1oxyl (3g). Yellow powder, yield 47\%, $0.149 \mathrm{~g}$. There are two peaks at the DSC curve on the heating run: $60{ }^{\circ} \mathrm{C}$ and $87^{\circ} \mathrm{C}$ and two peaks on the cooling run: $89^{\circ} \mathrm{C}, 46^{\circ} \mathrm{C}$. IR $\left(v_{\max }, \mathrm{cm}^{-1}\right)$ : $1728(\mathrm{C}=\mathrm{O}), 1606,1579(\mathrm{C}=\mathrm{N})$. ESR: $\mathrm{t}, \mathrm{A}_{\mathrm{N}} 1.455 \mathrm{mT}, \mathrm{g}_{\text {iso }} 2.0058$. Anal Calcd for $\mathrm{C}_{68} \mathrm{H}_{97} \mathrm{~N}_{2} \mathrm{O}_{7}$ (1054.51): C, 77.45; H, 9.27; N, 2.66\%. Found: C, 77.60; H, 9.31; N, 2.59\%.

\section{2,2-Dimethyl-8-(4-hydroxyphenyl)-3-[4-(4-(octyloxy)benzoyloxy)phenyl]-1,4-diazaspiro-}

[4,5]deca-3-en 1-oxyl (13). A solution of 4-octyloxybenzoic acid $(0.275 \mathrm{~g}, 1.1 \mathrm{mmol})$ in $1 \mathrm{ml}$ thionyl chloride was refluxed during $1.5 \mathrm{~h}$, excess of reagent was removed in vacuo. Residue was dissolved in dry THF $(10 \mathrm{ml})$ and solution was added dropwise to suspension of bis(phenole) 11 $(0.365 \mathrm{~g}, 1 \mathrm{mmol})$ and tert-BuOK $(0.123 \mathrm{~g}, 1.1 \mathrm{mmol})$ in $10 \mathrm{ml}$ of dry THF. The mixture was stirred at $\mathrm{rt}$ during $1 \mathrm{~h}$ and concentrated. Residue was treated with diethyl ether $(15 \mathrm{ml})$, washed by saturated aq solution of $\mathrm{NaHCO}_{3}(2 \times 8 \mathrm{ml})$ and water $(2 \times 8 \mathrm{ml})$. The organic layer was separated, dried over $\mathrm{MgSO}_{4}$ and the solvent evaporated. Residue was purified by CC (hexaneEtOAc, 4:1). Yellow powder, yield $28 \%, 0.167 \mathrm{~g}, \mathrm{mp} 165-166{ }^{\circ} \mathrm{C}(\mathrm{EtOH}) ; \mathrm{IR}\left(v_{\max }, \mathrm{cm}^{-1}\right): 3319$ $(\mathrm{OH}), 1718(\mathrm{C}=\mathrm{O}), 1602,1571(\mathrm{C}=\mathrm{N})$. ESR: t, $\mathrm{A}_{\mathrm{N}} 1.455 \mathrm{G}, \mathrm{g}_{\text {iso }}$ 2.0058. Anal. Calcd for $\mathrm{C}_{37} \mathrm{H}_{45} \mathrm{~N}_{2} \mathrm{O}_{5}$ (597.76): C, 74.34; H, 7.59; N, 4.69\%. Found: C, 74.45; H, 7.52; N, 4.71\%.

\section{2,2-Dimethyl-1-hydroxy-8-(4-hydroxyphenyl)-3-[4-(4-(octyloxy)benzoyloxy)phenyl]-1,4-}

diazaspiro[4,5] deca-3-en (14). A solution of $\mathrm{NH}_{4} \mathrm{Cl}(0.013 \mathrm{~g}, 0.24 \mathrm{mmol})$ in water $(0.1 \mathrm{ml})$ was added by syringe to a suspension of nitroxide $13(0.130 \mathrm{~g}, 0.22 \mathrm{mmol})$ and zinc dust $(0.029 \mathrm{~g}$, $0.44 \mathrm{mmol}$ ) in acetone $(3.5 \mathrm{ml})$. The mixture was stirred at $\mathrm{rt}$ during $1 \mathrm{~h}$. An inorganic precipitate was filtrated and the filtrate concentrated. Residue was treated with ethanol $(3 \mathrm{ml})$, precipitate formed was filtrated. White powder, yield $32 \%, 0.042 \mathrm{~g}, \mathrm{mp} 174-176{ }^{\circ} \mathrm{C}$; IR $\left(v_{\max }, \mathrm{cm}^{-1}\right): 1720$ $(\mathrm{C}=\mathrm{O}), 1608,1582(\mathrm{C}=\mathrm{N}) . \mathrm{NMR}{ }^{1} \mathrm{H}\left(400 \mathrm{MHz}, \mathrm{DMSO}-d_{6}\right): \delta_{\mathrm{H}} 0.85\left(3 \mathrm{H}, \mathrm{m}, \mathrm{CH}_{2} \mathrm{C}_{3}\right), 1.26$ $\left(10 \mathrm{H}, \mathrm{m}, 5 \mathrm{CH}_{2}\right), 1.42\left(6 \mathrm{H}, \mathrm{s}, \mathrm{Me}-11\right.$ and $\left.\mathrm{Me}-12+2 \mathrm{H}, \mathrm{m}, \mathrm{C}_{2}\right), 1.56(2 \mathrm{H}, \mathrm{m}, \mathrm{H}-6 e, 10 e), 1.75$ 
$(2 \mathrm{H}, \mathrm{m}, \mathrm{H}-7 e, 9 e), 1.93(2 \mathrm{H}, \mathrm{m}, \mathrm{H}-7 a, 9 a), 2.06(2 \mathrm{H}, \mathrm{m}, \mathrm{H}-6 a, 10 a), 2.47$ (1H, m, H-8a), 6.68 $\left(2 \mathrm{H}, \mathrm{d},{ }^{3} J_{3^{\prime \prime}, 2^{\prime \prime}} 8.04 \mathrm{~Hz}, \mathrm{H}-3^{\prime \prime}, 5^{\prime \prime}\right), 7.06\left(2 \mathrm{H}, \mathrm{d}, J_{2^{\prime \prime}, 3^{\prime \prime}} 8.04 \mathrm{~Hz}, \mathrm{H}-2^{\prime \prime}, 6^{\prime \prime}\right), 7.10\left(2 \mathrm{H}, \mathrm{d}, J_{3^{\prime}, 2^{\prime}} 8.55 \mathrm{~Hz}\right.$, H-3, $\left.5^{\prime}\right), 7.34\left(2 \mathrm{H}, \mathrm{d},{ }^{3} J_{3^{\prime \prime \prime}, 4^{\prime \prime \prime}} 7.48 \mathrm{~Hz}, \mathrm{H}-4^{\prime \prime \prime}, 6^{\prime \prime \prime}\right), 7.68(1 \mathrm{H}, \mathrm{s}, \mathrm{NOH}), 7.96\left(2 \mathrm{H}, \mathrm{d},{ }^{3} J_{4^{\prime \prime \prime}, 3^{\prime \prime \prime}} 7.48 \mathrm{~Hz}\right.$, H-3"', $\left.7^{\prime \prime \prime}\right), 8.08\left(2 \mathrm{H}, \mathrm{d},{ }^{3} J_{3^{\prime \prime}, 2^{\prime \prime}} 8.55, \mathrm{H}-3^{\prime \prime}, 5^{\prime \prime}\right), 9.11(1 \mathrm{H}, \mathrm{s}, \mathrm{OH}) . \mathrm{NMR}{ }^{13} \mathrm{C}\left(100 \mathrm{MHz}, \mathrm{DMSO}-d_{6}\right)$ : $\delta_{\mathrm{C}} 24.46(\mathrm{C}-11,12), 31.26$ (C-7, 9), 35.84 (C-6, 10), 42.37 (C-8), 69.61 (C-2), 88.97 (C-5), 114.70 (C-4"', 6"'), 114.98 (C-3", 5"), 120.61 (C-2'"), 122.06 (C-3', 5'), 127.44 (C-2", 6"), 129.03 (C 2', 6'), 130.90 (C-1'), 132.09 (C-3"', 7"'), 137.39 (C-1"), 152.18 (C-4'), 155.33 (C-4"), 163.96 (C-5'), 164.00 (C-1"') 171.70 (C-3). Alkyl chain signals: $13.21\left(\underline{\mathrm{CH}}_{3}\right), 22.12,25.44,28.52$, 26.67, 28.74, 31.51, $68.10\left(\mathrm{OCH}_{2}\right)$. Anal. Calcd for $\mathrm{C}_{37} \mathrm{H}_{46} \mathrm{~N}_{2} \mathrm{O}_{5}(598.77)$ : C, 74.22; H, 7.74; N, 4.68\%. Found: C, 74.12; H, 7.71; N, 4.65\%.

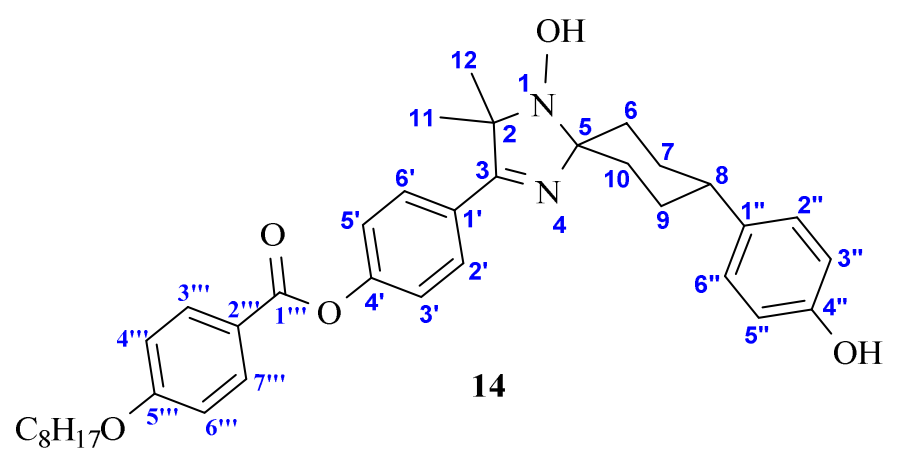

2,2-Dimethyl-8-[4-(4-nonyloxy)benzoyloxy)phenyl]-3-[4-(4-(octyloxy)benzoyloxy)phenyl]1,4-diazaspiro[4,5]deca-3-en 1-oxyl (4). A solution of 4-nonyloxybenzoic acid (0.079 g, 0.30 mmol) in $1 \mathrm{ml} \mathrm{SOCl} 2$ was refluxed during $1.5 \mathrm{~h}$, excess of reagent was removed in vacuo. Residue was diluted with dry THF $(10 \mathrm{ml})$ and added to suspension of nitroxide $\mathbf{1 3}(0.149 \mathrm{~g}$, $0.25 \mathrm{mmol})$ and triethylamine $(0.034 \mathrm{~g}, 0.3 \mathrm{mmol})$ in $10 \mathrm{ml}$ of dry THF. The mixture was stirred at $\mathrm{rt}$ during $1 \mathrm{~h}$ and concentrated. Residue was treated with diethyl ether $(15 \mathrm{ml})$, washed by saturated aq solution of $\mathrm{NaHCO}_{3}(2 \times 8 \mathrm{ml})$ and water $(2 \times 8 \mathrm{ml})$. The organic layer was separated, dried over $\mathrm{MgSO}_{4}$ and the solvent evaporated. Residue was purified by CC (hexane-EtOAc, 4:1). Yellow powder, $28 \%, 0.045 \mathrm{~g}, \mathrm{mp} 112-113^{\circ} \mathrm{C}(\mathrm{EtOH})$; IR $\left(v_{\max }, \mathrm{cm}^{-1}\right): 1732(\mathrm{C}=\mathrm{O}), 1606,1577$ $(\mathrm{C}=\mathrm{N})$. ESR: t, $\mathrm{A}_{\mathrm{N}} 1.455 \mathrm{mT}$, giso 2.0058. Anal. Calcd for $\mathrm{C}_{53} \mathrm{H}_{67} \mathrm{~N}_{2} \mathrm{O}_{7}$ (844.11): C, 75.41; $\mathrm{H}$, 8.00 ; N, 3.32\%. Found: C, 75.52; H, 7.94; N, 3.26\%.

\section{Acknowledgements}

This investigation was supported by joint research grant of RFBR-JSPS (project no. 11-0392107a), RFBR grant no. 13-03-00 427, Integration project SB RAS (no. 1) and Federal Agency for Education (agreement no.8456). The authors are also thankful to Dr. Vera D. Tikhova (NIOC SB RAS) and Dr. Katsuaki Suzuki (Kyoto University), for DSC measurements and polarizing microscope investigation of samples, respectively. 


\section{References}

1. Tebben, L.; Studer, A. Angew. Chem., Int. Ed. Engl. 2011, 50, 5034.

http://dx.doi.org//10.1002/anie.201002547

2. Hamada, S.; Furuta, T.; Wada, Y.; Kawabata, T. Angew. Chem., Int. Ed. Engl. 2013, 52, 8093.

http://dx.doi.org//10.1002/anie.201302261

3. Ratera, I.; Veciana, J. Chem. Soc. Rev. 2012, 41, 303. http://dx.doi.org//10.1039/C1CS15165G

4. Tretyakov, E. V.; Ovcharenko, V. I. Russ. Chem. Rev. 2009, 78, 971. http://dx.doi.org//10.1070/RC2009v078n11ABEH004093

5. Lahti, P. M. Magnetic Properties of Organic Materials; Marcel Dekker: New York, 1999.

6. Tamura, R.; Uchida, Y.; Suzuki, K. In Handbook of Liquid Crystals, Second Edition, eds. by Goodby, J. W.; Collings, P. J.; Kato, T.; Tschierske, C.; Gleeson, H. F.; Raynes, P., WileyVCH: Weinheim, 2014; Vol.8, pp 837-864.

7. Ikuma, N.; Tamura, R.; Shimono, S.; Kawame, N.; Tamada, O.; Sakui, N.; Yamauchi, J.; Yamamoto, Y. Angew. Chem., Int. Ed. Engl. 2004, 43, 3677.

http://dx.doi.org//10.1002/anie.200460007

8. Tamura, R.; Uchida, Y.; Ikuma, N. J. Mater. Chem. 2008, 18, 2872. http://dx.doi.org//10.1039/B802407C

9. Uchida, Y.; Ikuma, N.; Tamura, R.; Shimono, S.; Noda, Y.; Yamauchi, J.; Aoki, Y.; Nohira, H. J. Mater. Chem. 2008, 18, 2950. http://dx.doi.org//10.1039/B801704B

10. Uchida, Y.; Suzuki, K.; Tamura, R.; Ikuma, N.; Shimono, S.; Noda, Y.; Yamauchi, J. J. Am. Chem. Soc. 2010, 132, 9746. http://dx.doi.org//10.1021/ja101930d

11. Kogo, R.; Araoka, F.; Uchida, Y.; Tamura, R.; Ishikawa, K.; Takezoe, H. Appl. Phys. Expr. 2010, 3, 041701. http://dx.doi.org//10.1143/APEX.3.041701

12. Ikuma, N.; Tamura, R.; Shimono, S.; Uchida, Y.; Masaki, K.; Yamauchi, J.; Aoki, Y.; Nohira, H. Adv. Mater. 2006, 8, 477. http://dx.doi.org//10.1002/adma.200501531

13. Suzuki, K.; Uchida, Y.; Tamura, R.; Noda, Y.; Ikuma, N.; Shimono, S.; Yamauchi, J. Soft Matter. 2013, 9, 4687. http://dx.doi.org//10.1039/C3SM27295H

14. Jung, Y.-D.; Khan, M.; Park, S.-Y. J. Mater. Chem. B 2014, 2, 4922. http://dx.doi.org//10.1039/c4tb00476k

15. Hegmann, T.; Qi, H.; Marx, V. M. J. Inorg. Organomet. Polym. Mater. 2007, 17, 483. http://dx.doi.org//10.1007/s10904-007-9140-5 
16. Bisoyi, H. K.; Kumar, S. Chem. Soc. Rev. 2011, 40, 306.

http://dx.doi.org//10.1039/b901793n

17. Frach, R.; Tschierske, C.; Zaschke, H.; Deutscher, H-J. Liq. Cryst. 1989, 5, 197. http://dx.doi.org//10.1080/02678298908026363

18. Schmidt, W.; Vögtle, F.; Poetsch, E. Lieb. Ann. 1995, 1319. http://dx.doi.org//10.1002/jlac.1995199507175

19. Calaminus, W.; Vögtle, F.; Eidenschink, R. Z. Naturforsch 1986, 41b, 1011.

20. Boettcher, J.; Hartmann, R.; Vögtle, F. Chem. Ber. 1992, 125, 1865. http://dx.doi.org//10.1002/cber.19921250813

21. Poetsch, E.; Schmidt, W.; Vögtle, F.; Feuerbacher, N. DE 19755245, 1999; Chem. Abstr. 1999, 131, P65963j

22. Feuerbacher, N.; Vögtle, F.; Windscheidt, J.; Poetsch, E.; Nieger, M. Synthesis 1999, 117. http://dx.doi.org//10.1055/s-1999-3689

23. Miyazawa, K.; Yufit, D.S.; Howard, J.A.K.; de Meijere, A. Eur. J. Org. Chem. 2000, 4109. http://dx.doi.org//10.1002/1099-0690(200012)2000:24<4109::AID-EJOC4109>3.0.CO;2-Z

24. Miyazawa, K.; Demus, D.; de Meijere, A. Mol. Cryst. Liq. Cryst. 2001, 364, 253. http://dx.doi.org//10.1080/10587250108024994

25. Itoh, T.; Kanbara, M.; Nakajima, S.; Sakuta, Y.; Hayase, S.; Kawatsura, M.; Kato, T.; Miyazawa, K.; Uno, H. J. Fluor. Chem. 2009, 130, 1157. http://dx.doi.org//10.1016/j.fluchem.2009.06.007

26. Uchida, Y.; Matsuoka, N.; Takahashi, H.; Shimono, S.; Ikuma, N.; Tamura, R. Heterocycles 2007, 74, 607. http://dx.doi.org//10.3987/COM-07-S(W)38

27. Suzuki, K.; Mazhukin, D.G.; Takahashi, H.; Uchida, Y.; Tamura, R.; Grigor'ev, I. A. Heterocycles 2009, 78, 3091. http://dx.doi.org//10.3987/COM-09-11821

28. Zaytseva, E.V.; Gatilov, Yu. V.; Amitina, S.A.; Tamura, R.; Grigor`ev, I.A.; Mazhukin, D.G. Russ. J. Org. Chem. (Engl. Transl.) 2014, 50, 72. http://dx.doi.org//10.1134/S107042801401014X

29. Tamura, R.; Suzuki, K.; Uchida, Y.; Noda, Y. in Electron Paramagnetic Resonance, B.C. Gilbert, D.M. Murphy, V. Chechik (Eds.): RSC Publishing, 2013; Vol. 23, p.1. http://dx.doi.org//10.1039/9781849734837

30. Ionita, G.; Zarafu, I.; Paun, A.; Ionita, P. Mol. Cryst. Liq. Cryst. 2012, 562, 141. http://dx.doi.org//10.1080/15421406.2012.676834

31. Tschierske, C. Angew. Chem. Int. Ed. 2013, 52, 8828. http://dx.doi.org//10.1002/anie.201300872

32. Sevast'yanova, T.K.; Volodarskii, L.B. Bull. Acad. Sci. USSR, Div. Chem. Sci. (Engl. Trans.) 1972, 21, 2276. http://dx.doi.org//10.1007/BF00855320 
33. Reznikov, V.A.; Volodarskii, L.B. Chem. Heterocycl. Compd (Engl. Transl.) 1990, 643. http://dx.doi.org//10.1007/BF00756415

34. Hintermaier, F.; Volodarsky, L.B.; Polborn, K.; Beck, W. Liebigs Ann. Chem. 1995, 2189. http://dx.doi.org//10.1002/jlac.1995199512304

35. Reznikov, V. A.; Volodarsky, L. B. Russ. Chem. Bull. (Engl. Transl.) 1997, 46, 1577. http://dx.doi.org//10.1007/BF02502943

36. Volodarsky L.B. In Imidazoline Nitroxides: Synthesis and properties, Volodarsky, L. B. Ed., CRC Press: Boca Ratan, 1988; Vol. 1, 232.

37. http://limor1.nioch.nsc.ru/quant/conformers/shern/ld/

38. Graus, S.; Uriel, S.; Serrano, J. L. CrystEngComm 2012, 14, 3759. http://dx.doi.org//10.1039/c2ce06560f

39. Volodarsky, L. B.; Lapik A. S.; Russkikh, V. V.; Kobrin, V. S.; Lavretskaya, E. F.; Volkova, L. I.; Sarkisyan, D. A.; Borisov, M. M. SU 657016 (A1), 1979; Chem.Abstr. 1979, 91, P68767q.

40. Vasconcelos, U.; Dalmolin, E.; Merlo, A. Org. Lett. 2005, 7, 1027. http://dx.doi.org//10.1021/o1047524e

41. Impre, G.; Jakli, I.; Kalaszi, A.; Farkas, O. Advanced Automatic Generation of 3D Molecular Structures 1st European Chemistry Congress, Budapest, Hungary, August 27-31, 2006.

42. Chang, C.; Gilson, M.K. J. Comput. Chem. 2003, 24, 1987. http://dx.doi.org//10.1002/jcc.10325

43. O'Boyle, N.M.; Vandermeersch, T.; Flynn, Ch.J.; Maguire, A.R.; Hutchison, G. R. J. Cheminformatics 2011, 3, 8 . http://dx.doi.org//10.1186/1758-2946-3-8

44. Rocha, G.B.; Freire, R.O.; Simas, A.M.; Stewart, J.J.P. J. Comput. Chem. 2006, 27, 1101. http://dx.doi.org//10.1002/jcc.20425

45. MOPAC2012, James J. P. Stewart, Stewart Computational Chemistry, Colorado Springs, CO, USA, HTTP://OpenMOPAC.net (2012).

46. Perdew, J.P.; Burke, K.; Ernzerhof, M. Phys. Rev. Lett. 1996, 77, 3865.

http://dx.doi.org//10.1103/PhysRevLett.77.3865

47. Laikov, D. N. Chem. Phys. Lett. 2005, 416, 116.

http://dx.doi.org//10.1016/j.cplett.2005.09.046

48. http://www.nusc.ru 